\title{
Análise Fatorial Confirmatória do Instrumento de Avaliação Neuropsicológica Breve Infantil - NEUPSILIN - Inf
}

\author{
Jerusa Fumagalli de Salles - Universidade Federal do Rio Grande do Sul, Porto Alegre, Brasil \\ Juliana Burges Sbicigo - Universidade Federal do Rio Grande do Sul, Porto Alegre, Brasil \\ Wagner de Lara Machado - Universidade Federal do Rio Grande do Sul, Porto Alegre, Brasil \\ Monica Carolina Miranda - Universidade Federal de São Paulo, São Paulo, Brasil \\ Rochele Paz. Fonseca - Pontifícia Universidade Católica do Rio Grande do Sul, Porto Alegre, Brasil
}

\begin{abstract}
Resumo
Examinou-se a estrutura interna do Instrumento de Avaliação Neuropsicológica Breve Infantil - NEUPSILIN-Inf através de Análise Fatorial Confirmatória (AFC). Participaram do estudo 840 crianças (421 da cidade de São Paulo, 419 de Porto Alegre, Rio Grande do Sul), com idades entre seis e 12 anos $(\mathrm{M}=8,98, \mathrm{DP}=1,98)$, entre a primeira e a sexta série do ensino fundamental, de escolas públicas e privadas. Modelos teórico-hipotéticos foram testados considerando-se a organização original do instrumento e a literatura neuropsicológica. Verificou-se adequação empírica de cinco dos sete modelos inicialmente hipotetizados: praxias construtivas, memória verbal, memória episódica e semântica, processamento visual e desempenho acadêmico. Contrariando as hipóteses, linguagem ajustou-se em um modelo unifatorial, enquanto memória de trabalho, atenção e funções executivas foram mais bem explicadas por um modelo de dois fatores correlacionados. Concluiu-se que o NEUPSILIN-Inf apresenta evidência de validade de estrutura interna e pode ser utilizado na avaliação neuropsicológica de crianças para examinar esses grupos de componentes neurocognitivos.
\end{abstract}

Palavras-chave: Testes neuropsicológicos, Neuropsicologia, Cognição, Análise fatorial, Validade dos testes.

Confirmatory Factorial Analysis of the Child Brief Neuropsychological Assessment Battery - NEUPSILIN - Inf

\begin{abstract}
The aim of the study was to examine the internal structure of the Child Brief Neuropsychological Assessment Battery NEUPSILIN-Inf with Confirmatory Factor Analysis (CFA). The study included 840 children (421 in the city of São Paulo, 419 in Porto Alegre, Rio Grande do Sul), aged between six and 12 years ( $M=8.98, S D=1.98)$, between first and sixth grade in public and private schools. Theoretical and hypothetical models were tested considering the organization's original instrument and neuropsychological literature. There was empirical adequacy of five of the seven models initially hypothesized: constructive praxis, verbal memory, episodic and semantic memory, visual processing, and academic performance. Contrary to the hypothesis, language was fitted into a one-factor model, while working memory, attention and executive functions were best explained by a model of two correlated factors. We concluded that the NEUPSILIN-Inf presents evidence of validity as the internal structure and can be used in the neuropsychological evaluation of children to examine these groups of neurocognitive components.
\end{abstract}

Keywords: Neuropsychological tests, Neuropsychology, Cognition, Factor analysis, Validity of tests.

\section{Análisis Factorial Confirmatorio del Instrumento de Evaluación Neuropsicológica Breve Infantil - NEUPSILIN - Inf}

\section{Resumem}

Se examinó la estructura interna del Instrumento de Evaluación Neuropsicológica Breve Infantil - NEUPSILIN-Inf a través de Análisis Factorial Confirmatorio (AFC). Participaron del estudio 840 niños (421 de la ciudad de São Paulo, 419 de Porto Alegre, RS-Brasil), con edades entre seis y 12 años $(M=8,98, D E=1,98)$, entre el primer y sexto año de primaria, de escuelas públicas y privadas. Se testaron modelos teóricos hipotéticos considerándose la organización original del instrumento y la literatura neuropsicológica. Se verificó adecuación empírica de cinco de los siete modelos primeramente pensados: praxis constructivas, memoria verbal, memoria episódica y semántica, procesamiento visual y desempeño académico. Contrariamente a las hipótesis, el lenguaje se ajustó como un modelo de un factor, mientras memoria de trabajo, atención y funciones ejecutivas fueron más bien explicadas por un modelo de dos factores correlacionados. Se concluyó que el NEUPSILIN-Inf presenta evidencia de validez basada en la estructura interna y puede ser utilizado en la evaluación neuropsicológica de niños para examinar esos grupos de componentes neurocognitivos.

Palabras-clave: Pruebas neuropsicológicas, Neuropsicología, Cognición, Análisis factorial, Validez de las pruebas. 
A avaliação neuropsicológica consiste em um método utilizado para investigar se alterações cognitivas, acadêmicas, comportamentais e psiquiátricas são decorrentes de lesões, disfunções, doenças ou desenvolvimento anormal do cérebro (Semrud-Clikeman \& Ellison, 2009). Essa avaliação aplicada à neuropsicologia do desenvolvimento baseia-se no exame global das capacidades da criança, qualificando e quantificando as funções preservadas (potencialidades) e comprometidas (fragilidades), considerando a influência de aspectos socioemocionais (Ardila \& Rosselli, 2007). É fundamental na definição de quadros clínicos na infância, como dislexia e outras dificuldades de aprendizagem, transtorno de déficit de atenção e hiperatividade, transtornos de linguagem; traumatismos cranioencefálicos; epilepsias; transtornos globais do desenvolvimento, entre outros (Wright \& Sharples, 2008).

São objetivos da avaliação neuropsicológica infantil: identificar precocemente déficits ou dificuldades no desenvolvimento cognitivo e alterações no processo de aquisição de habilidades; examinar os efeitos dos déficits na capacidade de processar determinadas informações em domínios cognitivo-linguísticos específicos; e identificar possíveis áreas compensatórias executadas pelo cérebro visando a impulsionar as áreas comprometidas. Além disso, a avaliação neuropsicológica infantil visa à elaboração de programas de reabilitação a fim de promover o desenvolvimento das funções neuropsicológicas e/ou atenuar o impacto das dificuldades na aprendizagem e no comportamento (Semrud-Clikeman \& Ellison, 2009).

O desenvolvimento neuropsicológico infantil passa por diferentes processos de acordo com a maturação do sistema nervoso central (SNC) e sua interação dinâmica com experiências sociais, familiares e educacionais (Miranda \& Muszkat, 2004). A neuropsicologia infantil analisa cada etapa no desenvolvimento típico e nas situações nas quais ocorrem alterações funcionais ou estruturais no SNC, investigando a relação entre cérebro em desenvolvimento e comportamento infantil (Wright \& Sharples, 2008).

O paradigma clínico de avaliação neuropsicológica na infância envolve procedimentos de anamnese (com familiares ou responsáveis pela criança), observações clínicas, análise de exames complementares, administração de tarefas ecológicas, aplicação de escalas, testes e baterias neuropsicológicas, com características específicas às particularidades do desenvolvimento infantil (Strauss, Sherman \& Spreen, 2006). As baterias neuropsicológicas são especialmente importantes para discriminar entre funções comprometidas e preservadas nos transtornos de desenvolvimento, nos quais se observa uma sobreposição de dificuldades afetando mais de uma área cognitivo-linguística. É recomendada a utilização de procedimentos de rastreio em uma avaliação inicial e, uma vez identificados déficits, uma avaliação profunda com baterias completas que avaliem os processos cognitivo-linguísticos de interesse (Lezak, Howieson \& Loring, 2004).

No Brasil, uma importante limitação da avaliação neuropsicológica é a escassez de instrumentos internacionais padronizados para o português brasileiro e rigorosamente adaptados à realidade sociocultural e linguística do país (Fonseca \& cols., 2011). Em geral, os instrumentos são apenas traduzidos da língua inglesa, um procedimento inadequado, uma vez que palavras e imagens que são claras e comuns às crianças estrangeiras podem não ser comuns às crianças brasileiras (Anastasi \& Urbina, 2000). Nos últimos anos, contudo, esforços iniciais têm sido realizados para adaptação de baterias de avaliação infantil com destaque internacional, como é o caso da bateria NEPSY, avaliação neuropsicológica do desenvolvimento (NEPSY: developmental neuropsychological assessment, Korkman, Kirk \& Kemp, 1998) para crianças entre três e 12 anos de idade (Argollo \& cols., 2009) e de algumas escalas (funções visuais, linguagem receptiva e expressiva, escrita, leitura, aritmética e memória) da Bateria Neuropsicológica Luria-Nebraska para Crianças (Luria-Nebraska Neuropsychological Battery for Children - LNNB-C, Golden, 1987) entre oito e 12 anos (Crenitte, Batista, Silva, Lima \& Ciasca, 2011). Outra limitação à prática neuropsicológica infantil é a carência de baterias completas breves, isto é, que não exijam um tempo prolongado de aplicação e que sejam viáveis à utilização em serviços públicos de saúde. A bateria NEPSY, por exemplo, avalia cinco domínios cognitivos em duas ou três sessões de até 60 minutos, dependendo da idade da criança.

A fim de contribuir à redução dessas demandas, foi desenvolvido o Instrumento de Avaliação Neuropsicológica Breve Infantil - NEUPSILIN-Inf (Salles \& cols., 2011; no prelo), considerando as características socioculturais e linguísticas do Brasil, as particularidades do desenvolvimento infantil e os procedimentos para adaptação de instrumentos neuropsicológicos recomendados pela literatura internacional. A aplicação é realizada em uma única sessão com duração entre 40 e 60 minutos, dependendo da faixa etária. O NEUPSILIN-Inf inclui testes para avaliação de componentes de oito funções neuropsicológicas em crianças entre seis 
e 12 anos: orientação (temporal e espacial), atenção (visual e auditiva), percepção (visual e de emoções em faces), memória (episódico-semântica visuoverbal, episódico-semântica verbal, memória semântica e memória de trabalho), habilidades aritméticas, linguagem (oral e escrita), praxias construtivas e funções executivas. A linguagem é um dos construtos que perpassa a maioria dos subtestes, havendo tarefas específicas para avaliar o processamento da linguagem oral e escrita, além de veicular o exame das outras funções cognitivas por meio de estímulos verbais ou de visuais nomináveis. Desse modo, o instrumento é neuropsicolinguístico, podendo ser utilizado por profissionais/pesquisadores/clínicos que trabalham com desenvolvimento infantil. O objetivo é fornecer um perfil breve do funcionamento de processos neuropsicológicos (preservados e deficitários), que oriente a seleção de testes específicos a uma avaliação aprofundada.

Além de uma adequada adaptação sociocultural e linguística, um instrumento neuropsicológico deve ser submetido a estudos psicométricos visando à validade $\mathrm{e}$ fidedignidade por meio de uma série de procedimentos, alguns desses já analisados e descritos no manual do NEUPSILIN-Inf. (Salles \& cols., no prelo). A psicometria possui pressupostos teórico-técnicos que visam a assegurar a validade de construto mediante diferentes fontes de evidências, como a estrutura interna do instrumento, que pode ser verificada através de técnicas de análise fatorial (AERA, APA \& NCME, 1999). Dentre essas técnicas, a análise fatorial confirmatória (AFC) tem exercido significativa influência em estudos de validade de instrumentos por permitir testar hipóteses teóricas acerca dos padrões de intercorrelações entre as variáveis, ser eficaz para lidar com múltiplas relações e avaliar em detalhe os efeitos dos múltiplos erros de medida (Hair, Anderson, Tatham \& Black, 2009; Hershberger, 2003).

Em consulta às bases de dados internacionais ISI of Knowledge e PsycINFO, com os termos "neuropsychological assessment" e "factor analysis"/ "factor structure" (palavras-chave utilizadas no Thesaurus), identificaram-se duas baterias neuropsicológicas para crianças submetidas à análise fatorial, em um total de cinco estudos. Em dois deles, a estrutura interna da bateria NEPSY: Developmental Neuropsychological Assessment (Korkman \& cols., 1998) foi investigada com AFC (Mosconi, Nelson \& Hooper, 2008) e análise fatorial exploratória (AFE) (Stinnett, Oehler-Stinnett, Fuqua \& Palme, 2002). Em três estudos, as baterias Reitan-Indiana Neuropsychological Battery para crianças mais jovens e Halstead-Reitan Neuropsychological Test Battery for Older Children (Reitan \& Wolfson, 1992) foram submetidas ao método de componentes principais (Krug, Dean \&Anderson, 1995; Livingston, Gray, Haak \& Jennings, 2000; Livingston, Gray, Haak \& Jennings, 1997). Estudos dessa natureza não foram encontrados na literatura nacional, conforme busca na base de dados BVS-PSI, com os termos "testes neuropsicológicos" e "análise fatorial” (Terminologia em Ciências da Saúde, DeCS). Fazem-se necessários, portanto, estudos brasileiros investigando a validade de estrutura interna de baterias neuropsicológicas infantis.

Desse modo, o objetivo do presente estudo foi investigar, com análise fatorial confirmatória (AFC), a estrutura interna do NEUPSILIN-Inf. Com base no modelo teórico-hipotético do instrumento (sua organização em oito domínios cognitivos) e na literatura neuropsicológica, foram formulados sete modelos teóricos e submetidos a confirmação empírica: 1) linguagem, consistindo de dois fatores correlacionados - linguagem oral e escrita - compostos pelas tarefas designadas para essas duas funções e também pela tarefa de span de pseudopalavras (tarefa de memória de trabalho), por também ser considerada uma tarefa de linguagem oral; 2) praxias construtivas; 3 ) memória de trabalho, atenção e funções executivas em um modelo de três fatores correlacionados, uma vez que há interdependência entre esses processos cognitivos; 4) memória verbal, composto por tarefas de span de memória de trabalho, evocação imediata e tardia, memória semântica e orientação, 5) memória episódico-semântica, com tarefas de evocação imediata, memória semântica e orientação; 6) processamento visual, reunindo tarefas com estímulos visuais e 7) desempenho acadêmico, com tarefas envolvendo habilidades de leitura, escrita e aritméticas.

\section{Método}

Participaram 840 crianças, com idades entre seis e 12 anos, cursando entre o $1^{\circ}$ ano e a $6^{a}$ série do ensino fundamental. Do total, 421 (50,4\% meninas, $\left.\mathrm{M}_{\text {idade }}=8,98, \mathrm{DP}=1,99\right)$ pertenciam a 14 escolas (seis públicas) da cidade de São Paulo/SP, com 50,2\% frequentando escola pública, enquanto 419 (54,4\% meninas, $\left.\mathrm{M}_{\text {idade }}=8,99, \mathrm{DP}=1,97\right)$ pertenciam a 16 escolas (sete públicas) da cidade de Porto Alegre/RS, com $50,8 \%$ frequentando a escola pública. Os critérios de inclusão foram ausência de histórico de doenças neurológicas ou psiquiátricas, dificuldades auditivas e/ 
ou visuais não corrigidas (questionário preenchido pelos pais/ responsáveis), dificuldades de aprendizagem e repetência escolar (questionário preenchido pela professora); ausência de indicadores de alterações comportamentais e de transtorno de déficit de atenção e hiperatividade, conforme Escala Conners Abreviada para Professores (Brito, 1987). As crianças apresentaram percentil superior a 25 na amostra do RS e na amostra de SP, percentil superior 20 no Teste Matrizes Progressivas de Raven (Raven, Court \& Raven, 1986), conforme tabela normativa (Angelini, Alves, Custódio, Duarte \& Duarte, 1999).

\section{Instrumento}

O NEUPSILIN-Inf (Salles \& cols., 2011; no prelo) inclui diferentes tipos de tarefas, que serão apresentadas com seu respectivo índice de confiabilidade $(\alpha$.$) quando suas características (ex.: permitir variabili-$ dade de respostas) possibilitaram a estimação do índice. $\mathrm{O}$ instrumento possui tarefas de orientação temporal e espacial ( 6 pontos, $\alpha .=0,66)$; percepção de emoções em faces ( 2 pontos) e de constância de forma e de objeto (4 pontos); atenção visual pelo teste de cancelamento de figuras (35 pontos) e pela tarefa de span de dígitos na ordem direta (24 pontos, $\alpha .=0,63)$. Memória de trabalho ( 96 pontos, $\alpha .=0,82)$ envolve os subtestes de span de pseudopalavras (20 pontos, $\alpha .=0,60)$ e span de dígitos na ordem inversa ( 28 pontos, $\alpha .=0,70$ ), que avaliam os componentes fonológico e executivo central (somam 48 pontos). O componente visuoespacial é investigado por uma tarefa semelhante aos blocos de Corsi, mas bidimensional (28 pontos, $\alpha .=0,72$ ). A memória verbal inclui tarefas de evocação imediata e tardia de lista de palavras ( 9 pontos por tarefa), e a memória episódica visuoverbal inclui evocação imediata de lista de figuras (9 pontos). Quatro questões acessam o conhecimento prévio da criança na tarefa de memória de longo prazo semântica (4 pontos). No escore total de linguagem (73 pontos, $\alpha .=0,94)$, linguagem oral (32 pontos, $\alpha .=0,71)$ e linguagem escrita (41 pontos, $\alpha .=0,92)$ são avaliadas por meio dos subtestes: nomeação de figuras ( 9 pontos); consciência fonológica (10 pontos); compreensão oral de palavras e frases (5 pontos) e processamento inferencial (8 pontos); leitura em voz alta de sílabas, palavras e pseudopalavras (17 pontos); compreensão escrita de palavras e frases (5 pontos); escrita sob ditado de palavras e pseudopalavras (19 pontos); escrita espontânea e escrita copiada de frases (escore varia de zero a dois pontos em cada tarefa). Habilidades visuoconstrutivas são investigadas através da cópia de quatro figuras apresentadas individualmente (24 pontos, $\alpha .=0,80)$. Habilidades aritméticas são mensuradas pelos subtestes contagem de palitos e cálculos matemáticos das quatro operações ( 25 pontos, $\alpha .=0,90)$. Funções executivas são avaliadas por tarefas de fluência verbal (ortográfica e semântica), cujo escore é o número de palavras evocadas, e tarefa go/no go auditiva (60 pontos).

\section{Procedimentos}

O projeto foi aprovado pelo Comitê de Ética em Pesquisa do Instituto de Psicologia da UFRGS (protocolo de número 2008/067). Após assinatura do Termo de Consentimento Livre e Esclarecido, os pais preencheram o Questionário de Dados Sociodemográficos e de Saúde. A avaliação das crianças ocorreu em duas sessões, sendo a primeira coletiva, de aproximadamente 20 minutos, e outra individual, de aproximadamente 60 minutos. O Teste de Raven - Escala Especial (Angelini \& cols., 1999) foi aplicado coletivamente (máximo oito estudantes), na maioria das crianças, segundo disponibilidade de sala e frequência de alunos nos dias de coleta. O segundo encontro foi individual, para aplicação do NEUPSILIN-Inf.

\section{Análise dos dados}

Foram realizadas análises descritivas das variáveis, com a finalidade de explorar suas características de distribuição (assimetria, curtose e frequência). Para investigar a adequação dos dados amostrais em relação aos modelos teóricos propostos no presente estudo, foram conduzidas análises fatoriais confirmatórias (AFC's) por meio do software Structural Equations (EQS, v.6.1, Bentler, 2004). A matriz de variância/covariância foi submetida ao método de estimação máxima verossimilhança (maximum likelihood) e os seguintes índices de ajuste (valores de referência) foram utilizados para interpretação dos modelos: Comparative Fit Index (CFI $\geq 0,90)$, Non Normed Fit Index (NNFI $\geq 0,90)$ e Standardized Root Mean Residual (SRMR $\leq 0,08)$ (Schreiber, Stage, King, Nora \& Barlow, 2006). Os índices CFI e NNFI contrastam a matriz de dados amostrais com um modelo nulo ou de independência, ao passo que o índice SRMR contrasta essa mesma matriz com um modelo saturado. O índice Consistent Akaike Information Criterion (CAIC) foi utilizado na comparação de modelos concorrentes, quando necessário. Esse índice não possui valores de referência, valores menores favorecendo o modelo de melhor ajuste e valores de $\Delta C A I C>10$ indicando diferenças significativas entre os modelos (Akaike, 1987). A fidedignidade de cada fator foi estimada por meio do 
índice de fidedignidade composta, sendo considerados adequados valores $>0,60$ (Hair \& cols., 2009).

\section{Resultados}

As análises descritivas evidenciaram a presença de efeito de teto nas variáveis orientação, cancelamento de figuras (atenção visual), percepção de emoção em faces, constância de forma, constância de objeto (ambos de percepção visual), nomeação de figuras e compreensão oral de palavras e sentenças (linguagem oral), contagem de palitos (habilidades aritméticas) e escrita copiada de uma frase (linguagem escrita) (resultados não exibidos). Percepção de emoção em faces, constância de forma,

M1b

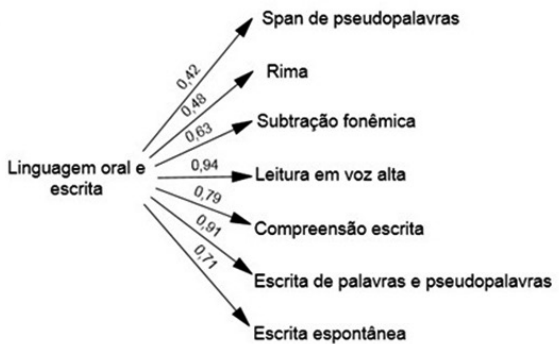

M3b

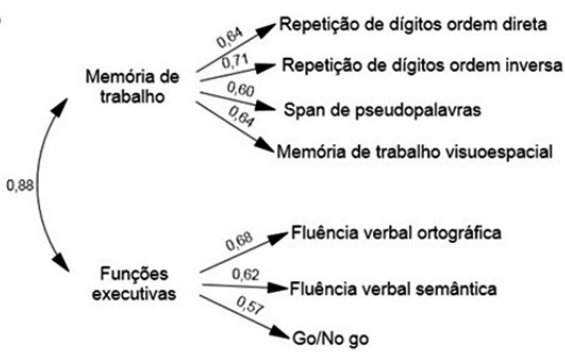

M5

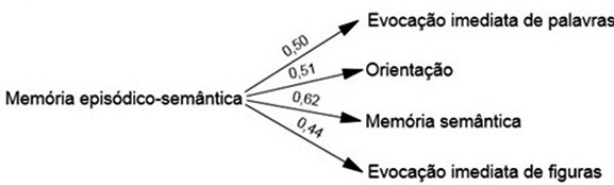

M7

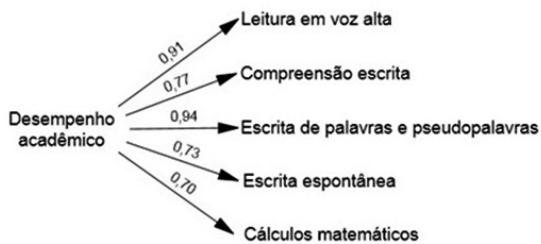

constância de objeto e compreensão oral são subtestes avaliados por tarefas de múltipla escolha, o que reduz a variabilidade de escores e dá margem para acertos ao acaso (por exemplo, nas tarefas de constância de forma e constância do objeto há $50 \%$ de chance de indicar a resposta certa). Essas variáveis foram inicialmente incluídas na estimação de seus respectivos modelos, contudo, por causa da pouca variabilidade e da penalização dos modelos em relação aos índices de ajuste, apenas as variáveis orientação e cancelamento de figuras foram mantidas nas análises posteriores, uma vez que contribuíram para alguns modelos.

Os sete modelos inicialmente formulados foram submetidos à confirmação empírica: 1) linguagem oral e escrita (dois fatores correlacionados), 2) praxias

M2

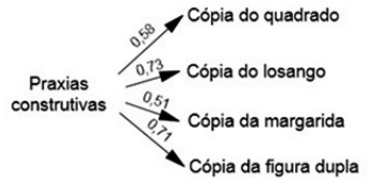

M4

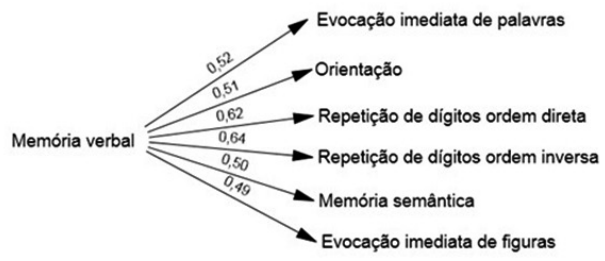

M6

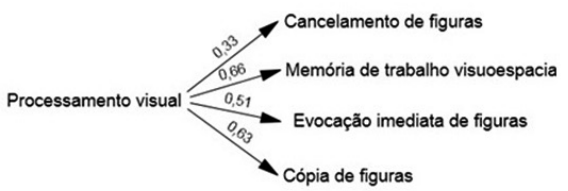

Figura 1. Modelos teórico-hipotéticos de linguagem, memória de trabalho e funções executivas, memória verbal, praxias, construtivas, processamento visual e desempenho acadêmico. As covariâncias entre os fatores $(\varphi)$ e cargas fatoriais dos itens $(\lambda)$ são estatisticamente diferentes de zero, ao nível de significância $p>0,001$ 
construtivas (unifatorial), 3) memória de trabalho, atenção e funções executivas (três fatores correlacionados), 4) memória verbal (unifatorial, composto por tarefas de span de memória de trabalho, evocação imediata e tardia, memória semântica e orientação), 5) memória episódico-semântica (unifatorial, composto por tarefas de evocação imediata, memória semântica e orientação); 6) processamento visual (unifatorial, reunindo as tarefas que envolvem estímulos visuais), e 7) desempenho acadêmico (unifatorial, com tarefas envolvendo habilidades de leitura, escrita e habilidades aritméticas).

$\mathrm{Na}$ Figura 1, são observadas as variáveis que compuseram cada modelo, valores de covariância entre os fatores $(\varphi)$ e as cargas fatoriais dos itens $(\lambda)$. O modelo de linguagem foi testado a partir da hipótese de um modelo de dois fatores correlacionados (linguagem oral e escrita) seguindo a organização original do NEUPSILIN-Inf, com acréscimo da tarefa de span de pseudopalavras ao fator linguagem oral. Como se pode verificar na Tabela 1, o referido modelo (M1a) apresentou índices de ajuste adequados. Contudo, os dois fatores mostraram-se altamente correlacionados $(>0,9)$, sugerindo que poderiam ser condensados em um fator. Além disso, a confiabilidade do fator linguagem oral situou-se um pouco abaixo do ponto crítico 0,57 . Desse modo, foi testado um modelo unifatorial (M1b) de linguagem, que se mostrou ajustado e com alta confiabilidade (ver Tabela 1). Uma vez que o modelo M1a não mostrou uma vantagem robusta quanto aos indicadores de ajuste em relação ao modelo M1b, optou-se por esse último por ser mais parcimonioso. Não houve contribuição dos subtestes de compreensão oral (palavras e sentenças, opção de múltipla escolha), processamento de inferências e escrita copiada (efeito de teto). Assim, linguagem oral (incluindo habilidades metalinguísticas) incluiu tarefas de habilidades de processamento fonológico da linguagem (consciência fonológica - subtestes de rima e subtração fonêmica e memória fonológica - adicionando-se o subteste de span de pseudopalavras). Linguagem escrita manteve quatro de seus cinco subtestes originais: leitura em voz alta, compreensão escrita, escrita de palavras e pseudopalavras, e escrita espontânea (ver Figura 1).

O modelo praxias construtivas (M2) também obteve ajuste adequado aos dados (ver Tabela 1) e manteve seus respectivos itens (um único subteste). $\mathrm{O}$ modelo de três fatores correlacionados (M3a), memória de trabalho (span de dígitos na ordem inversa, span de pseudopalavras e memória de trabalho visuoespacial), atenção (cancelamento de figuras e span de dígitos na ordem direta) e funções executivas (tarefas go/no go auditiva, fluência verbal ortográfica e semântica), também foi ajustado (ver Tabela 1), contudo a correlação entre os fatores foi muito alta $(>0,9)$, além do que a tarefa de cancelamento de figuras não contribuiu para o construto atenção. Desse modo, foram testados dois modelos adicionais: modelo geral de funções executivas (incluindo os subtestes dos três domínios em um único fator, M3b) e modelo de dois fatores correlacionados (memória de trabalho e funções executivas, M3c). Na Tabela 1, verifica-se que o modelo M3b obteve índices de ajuste inferior aos dos modelos M3a e M3c. O modelo M3c, na comparação com o modelo M3a, mostrou um índice CAIC menor $(\Delta C A I C>10)$. Desse modo, o modelo M3c foi considerado mais parcimonioso, no qual a tarefa de atenção span de dígitos na ordem direta foi realocada no fator de memória de trabalho (correspondendo ao componente fonológico do modelo de memória de trabalho).

Os modelos de memória verbal (M4), memória episódico-semântica (M5), processamento visual (M6) e desempenho acadêmico (M7) foram formulados com subtestes de diferentes domínios cognitivos do instrumento teoricamente coerentes e submetidos à confirmação empírica. Memória verbal (M4) englobou memória episódica com os subtestes de evocação imediata de listas de palavras (a evocação tardia dessa mesma lista foi inicialmente incluída no modelo, porém estava muito correlacionada à evocação imediata e, então, foi retirada) e de lista de figuras, assim como memória de trabalho com os subtestes de evocação imediata de sequências de dígitos na ordem direta e inversa (span), tarefas clássicas na literatura de memória verbal (Strauss \& cols., 2006). Além disso, englobou o subteste de memória semântica e as tarefas de orientação espacial e temporal, que envolvem conhecimentos prévios e conhecimento geral (inclusive memória semântica), tendo apresentado bom ajuste ao modelo. O modelo de memória episódicosemântica (M5) foi formulado mantendo as tarefas do modelo anterior, com exceção das tarefas de memória de trabalho, para refletir somente os componentes de memória episódica e semântica do instrumento. Os índices de ajuste dos dois modelos descritos (memória verbal e memória episódico-semântica) foram adequados, contudo a confiabilidade do modelo de memória verbal foi mais alta, enquanto a de memória episódicosemântica foi ligeiramente abaixo do ponto crítico (ver Tabela 1). 
Os subtestes que exigiam inicialmente a percepção e processamento visual do estímulo (verbal ou não verbal) foram adequadamente ajustados em um modelo de processamento visual (M6), que incluiu a tarefa de cancelamento de figuras (único modelo para o qual essa tarefa contribuiu), memória de trabalho visuoespacial, evocação imediata e tardia de figuras e cópia de figuras (subteste de praxias construtivas). O modelo de desempenho acadêmico (M7) também obteve índices de ajuste satisfatórios, com as tarefas de leitura em voz alta, compreensão escrita, escrita de palavras e pseudopalavras, escrita espontânea e cálculos matemáticos.

A Tabela 1 apresenta os índices de ajuste para cada modelo investigado e medidas de fidedignidade composta para cada fator. Em geral, os índices de ajuste (CFI, NNFI e SRMR) situaram-se dentro dos valores de referência, indicando a adequação dos dados amostrais para reproduzir os modelos teóricos investigados (ver Figura 1).

\section{Discussão}

Neste estudo, buscou-se verificar evidências de validade de estrutura interna para o NEUPSILIN-Inf por meio da técnica de AFC. Dos sete modelos teóricos inicialmente formulados, cinco foram confirmados: praxias construtivas, memória verbal, memória episódico-semântica, processamento visual e desempenho acadêmico. Os outros dois modelos formulados - linguagem oral e escrita (dois fatores correlacionados) e memória de trabalho, atenção e funções executivas (três fatores correlacionados) - não foram adequados em razão da alta correlação entre os fatores. Ainda, alguns subtestes não contribuíram para esses modelos, como no caso de algumas tarefas de linguagem oral e da tarefa de cancelamento (atenção visual), que não contribuiu para atenção. Assim, um modelo unifatorial foi suficiente para explicar o construto linguagem, enquanto um modelo de dois fatores correlacionados foi o mais adequado para memória de trabalho e funções executivas.

Alguns subtestes (cópia de figuras, escrita de palavras e pseudopalavras, escrita espontânea, evocação imediata de figuras, compreensão escrita, leitura em vOZ alta, span de dígitos na ordem direta e na ordem inversa, span de pseudopalavras e MT visuoespacial) adequaramse a mais de um construto neuropsicológico, revelando a interdependência dos processos cognitivos avaliados, como já havia sido previsto na formulação dos modelos. Sabe-se que um subteste não pode examinar apenas

Tabela 1. Modelos Investigados, Índices de Ajuste pelo Método de Estimação Máxima Verossimilhança e Índices de Fidedignidade Composta

Modelos investigados

M1a. Linguagem oral e escrita

M2. Praxias construtivas

M3a. Memória de trabalho, atenção e funções executivas

M3b. Funções executivas global

M3c. Memória de trabalho e funções executivas

M4. Memória verbal

M5. Memória episódico-semântica

M6. Processamento visual

M7. Desempenho acadêmico
Índices de ajuste

\begin{tabular}{cccc} 
CFI & NNFI & SRMR & FC \\
\hline 0,99 & 0,98 & 0,018 & $\begin{array}{c}\text { LO }=0,57 \\
\text { LE }=0,91\end{array}$ \\
0,98 & 0,97 & 0,023 & 0,88 \\
0,99 & 0,96 & 0,023 & 0,73 \\
0,93 & 0,89 & 0,041 & MT $=0,74$ \\
& & & AT $=0,24$ \\
& & & FE $=0,65$ \\
0,92 & 0,87 & 0,048 & 0,81 \\
0,93 & 0,90 & 0,046 & MT $=0,74$ \\
& & & FE $=0,65$ \\
0,96 & 0,94 & 0,033 & 0,72 \\
0,96 & 0,89 & 0,030 & 0,59 \\
1,00 & 1,00 & 0,007 & 0,62 \\
0,96 & 0,91 & 0,032 & 0,91 \\
\hline
\end{tabular}

Nota. $\mathrm{M}=$ memória de trabalho, $\mathrm{FE}=$ =funções executivas, $\mathrm{LO}=$ linguagem oral, $\mathrm{LE}=$ linguagem escrita, $\mathrm{CFI}=$ Comparative Fit $\mathrm{Index}, \mathrm{NNFI}=\mathrm{Non}$ -

Normed Fit Index, SRMR=Standardized Root Mean Residual, FC=índice de fidedignidade composta. 
um componente cognitivo, portanto, alguns subcomponentes participam da demanda de diferentes tarefas (Simões, 2002).

A adequação de um subteste a modelos diferentes corrobora um princípio importante da avaliação neuropsicológica, ou seja, de que uma tarefa dificilmente analisará uma habilidade isolada. Os resultados dos testes são multideterminados, envolvendo uma diversidade de processos neuropsicológicos, e vários desses processos podem contribuir para o desempenho num único teste. A linguagem, por exemplo, perpassa a maioria, senão todas as tarefas do instrumento, pois os itens-estímulos em geral são verbais e as respostas, na maioria das vezes (com exceção das de múltipla escolha e de cancelamento), são orais (Fonseca \& cols., 2011; Simões, 2002). A contribuição de subtestes a diferentes modelos reflete uma das características específicas da análise fatorial aplicada a instrumentos neuropsicológicos, pois isso não deve ocorrer quando a técnica é aplicada a testes psicológicos que buscam a delimitação de fatores totalmente independentes. A psicometria exerce papel diferencial em medidas neuropsicológicas, uma vez que o desempenho observado é relacionado ao funcionamento cognitivo geral (Fonseca \& cols., 2011). Além disso, as tarefas são comparadas para a interpretação de funções cognitivas preservadas, deficitárias ou menos estimuladas. Portanto, as tarefas ajustam-se a diferentes modelos por recrutarem processos que podem não ser passíveis de separação (Simões, 2002).

$\mathrm{Na}$ tarefa de span de pseudopalavras, há envolvimento de memória de trabalho e linguagem oral (processamento fonológico), pois as pseudopalavras são armazenadas na memória por curto prazo e repetidas oralmente. $\mathrm{Na}$ tarefa span de dígitos na ordem direta e na ordem inversa há envolvimento de memória de trabalho (componentes fonológico e executivo central, respectivamente). Span de dígitos na ordem inversa foi a tarefa que mais contribuiu para o fator de memória de trabalho, possivelmente por recrutar o componente executivo central (manter a sequência de dígitos original, inverter mentalmente e responder oralmente à nova ordem). A tarefa de memória de trabalho visuoespacial (reprodução inversa) envolve os componentes executivo central e visuoespacial da memória de trabalho, e processamento visual. De forma similar, é esperado o envolvimento do processamento visual e de praxias construtivas no subteste de cópia de figuras (que é o escore total dos itens do modelo de praxias, M2, Figura 1).
As tarefas de atenção foram ajustadas em modelos de outras funções neuropsicológicas. Span de dígitos na ordem direta contribuiu para o componente fonológico da memória de trabalho, enquanto cancelamento de figuras, para o modelo de processamento visual. Cabe salientar que o instrumento não avalia, de forma independente, o construto atenção nas suas diferentes modalidades (sustentada, dividida, alternada). De forma similar, o construto funções executivas, conforme esperado pelos autores, não contempla todos os subprocessos das funções executivas frias, mais ligadas ao raciocínio (Verdejo-García \& Bechara, 2010), com tarefas de planejamento, monitoramento, entre outras. Os subtestes incluídos envolvem componentes executivos de inibição e flexibilidade cognitiva, pelos subtestes fluência verbal (também pode ser de linguagem - acesso lexical) e go/ no go.

Contrariando a hipótese inicial, os subtestes de linguagem ajustaram-se melhor em um modelo unifatorial, o que deve ter sido influenciado pelo fato de que algumas tarefas de linguagem oral foram excluídas da análise devido ao efeito de teto. $\mathrm{O}$ modelo unifatorial incluiu somente três tarefas de linguagem oral, sugerindo que essa habilidade precisa ser complementada com outros instrumentos específicos, além do NEUPSILIN-Inf, para a investigação de componentes linguísticos, como elementos sintáticos e semânticos no nível de sentenças e de texto. O componente pragmático (tarefa de processamento de inferências) está contemplado no instrumento, porém não se ajustou ao modelo, possivelmente porque essa tarefa está mais relacionada a aspectos funcionais da linguagem, com contribuição do hemisfério direito, enquanto as demais tarefas do modelo estão mais relacionadas a aspectos estruturais da linguagem, com contribuição do hemisfério esquerdo (Beeman, 1998). Linguagem escrita está contemplada pelo modelo com todos os subtestes previstos, exceto escrita copiada de uma sentença. Já seria esperado que esse último subteste não se ajustasse ao modelo, tanto pela baixa variabilidade de escores quanto por envolver mais habilidades perceptovisuais e praxias do que linguagem propriamente dita. A condensação das tarefas de linguagem oral e escrita em um único fator também ilustra a sua estreita relação. Linguagem oral (incluindo consciência fonológica, léxico, morfemas, sintaxe, estrutura e pragmática) é considerada uma sólida fundação para o progresso da linguagem escrita. Ambas compartilham um conjunto de habilidades cognitivas, como memória de trabalho, coesão linguística e conhecimento morfológico (Beeman, 1998; Strauss e cols., 2006).

Psico-USF, Bragança Paulista, v. 19, n. 1, p. 119-130, jan. / abril 2014 
O modelo de praxias construtivas também apresentou adequação empírica. Os quatro itens que compõem o modelo (cópia de figuras) são direcionados a avaliar a capacidade visuoconstrutiva da criança, que exige habilidades visuais e de planejamento motor (Semrud-Clikeman \& Ellison, 2009). Com relação aos subtestes de atenção, memória de trabalho e funções executivas, estes se ajustaram em um modelo de dois fatores correlacionados, ao invés de três, contrariando a hipótese inicial. Esse resultado é influenciado pela forma de avaliar a atenção no instrumento, ou seja, com dois subtestes, um deles não contribuiu para o modelo (cancelamento de figuras) e o outro foi direcionado à avaliação do componente fonológico de memória de trabalho. Memória de trabalho é o sistema responsável pela manutenção ativa e manipulação da informação durante breves períodos de tempo (Baddeley \& Hitch, 1974). O modelo teórico mais influente é o multicomponencial de Baddeley (Baddeley \& Hitch, 1974; Baddeley e cols., 2011), que divide o sistema nos componentes fonológico, visuoespacial e executivo central, além do buffer episódico (a avaliação do buffer não foi diretamente incluída na construção das tarefas de memória de trabalho do NEUPSILIN-Inf). O executivo central é responsável por controlar o processamento da informação na memória de trabalho, direcionando a atenção, mantendo objetivos das tarefas, tomada de decisão e recuperação da memória (Baddeley e cols., 2011). Esse conceito agrega-se ao de funções executivas, que são processos cognitivos complexos necessários à organização e adaptação do comportamento a um ambiente em constante mudança. Incluem habilidades como inibição, planejamento, flexibilidade mental, fluência verbal e memória de trabalho (Lezak \& cols., 2004). A alta correlação entre esses construtos (ver Figura 1), observada neste estudo, corrobora a literatura (Baddeley e cols., 2011; McCabe, Roediger, McDaniel, Balota \& Hambrick, 2010) sugerindo que essas funções podem ser empiricamente distinguidas ainda que compartilhem características por associações cognitivas de seus subcomponentes. Essa alta correlação ocorre porque as tarefas de memória de trabalho e de funções executivas devem compartilhar um componente de atenção executiva preditivo da cognição de alto nível (McCabe e cols., 2010).

O NEUPSILIN-Inf também avalia memória verbal com as tarefas de memória episódica, responsável pelo armazenamento de eventos específicos; memória semântica, responsável pelo armazenamento de conhecimento factual que independe da experiência pessoal (Tulving \& Szpunar, 2009), orientação espacial (envolve componente episódico) e span de dígitos de memória de trabalho. A memória episódica permite que uma lembrança tome a forma de uma "viagem" mental no tempo subjetivamente experenciado. Diferente da memória semântica, ela é autocentrada (Tulving \& Szpunar, 2009). O desenvolvimento ontogenético da memória episódica é anterior ao da semântica, a primeira possibilita a formação da segunda e essa última facilita a formação de novos episódios (Scarf, Gross, Colombo \& Hayne, 2011).

As habilidades avaliadas pelos subtestes do modelo de processamento visual são necessárias à codificação das informações perceptuais visuais na memória de trabalho e, assim, à aprendizagem e retenção dessas informações na memória de longo prazo. Sabe-se que outros processos estão envolvidos na execução dos subtestes visuais, uma vez que alguns estímulos podem ser nomináveis e envolvem a integração com habilidades motoras (Baron, 2004). Dificuldades no processamento visual resultam em problemas para reconhecer, organizar, interpretar ou recordar letras, palavras, números, formas, prejudicando o progresso escolar (McIntosh, 2007). Já o modelo de desempenho acadêmico inclui medidas de desempenho utilizadas na avaliação neuropsicológica infantil, com a avaliação de habilidades de leitura, conhecimentos gerais, escrita e aritmética (Semrud-Clikeman \& Ellison, 2009). Embora as tarefas do NEUPSILIN- Inf não avaliem essas habilidades de forma aprofundada, elas servem de triagem para indicar a necessidade de maior investigação e/ou intervenção especial (Strauss \& cols., 2006).

É limitação do presente estudo o uso de AFC em um instrumento breve, que possui poucos itens em alguns de seus subtestes. $\mathrm{O}$ fato de que algumas tarefas apresentaram efeito de teto também prejudicou a análise, como, por exemplo, no caso do modelo de linguagem que foi composto predominantemente por tarefas de linguagem escrita em virtude do efeito de teto em algumas tarefas de linguagem oral. Apesar disso, os resultados apontam que o instrumento possui validade quanto à estrutura interna para os modelos de funções neuropsicológicas confirmados.

Estudos em andamento estão buscando evidências de validade do NEUPSILIN-Inf, com base em variáveis externas, incluindo a validade clínica através de estudos com crianças com patologias (p. ex.: dificuldades de leitura), analisando-se, ainda, se tarefas com efeito de teto em crianças em desenvolvimento típico podem diferenciar grupos clínicos e não clínicos. Pesquisas 
futuras deverão buscar indícios de sensibilidade e especificidade do NEUPSILIN-Inf. O instrumento pode ser muito promissor no início do processo diagnóstico e prognóstico de diferentes fases de desenvolvimento típico e atípico, em crianças com quadros neurológicos e/ou psiquiátricos com sequelas neurocognitivas.

\section{Referências}

Akaike, H. (1987). Factor analysis and AIC. Psychometrika, 52, 317-332.

American Educational Research Association, American Psychological Association, \& National Council on Measurement in Education (1999). Standards for Educational and Psychological Testing. Washington, DC: AERA, 1999.

Anastasi, A., \& Urbina, S. (2000). Testagem psicológica. Porto Alegre: Artes Médicas.

Angelini, A., Alves, I., Custódio, E., Duarte, W., \& Duarte, J. (1999). Matrizes Progressivas Coloridas de Raven. Manual. São Paulo, SP: Centro Editor de Testes e Pesquisa em Psicologia.

Ardila, A., \& Rosselli, M. (2007). Neuropsicología clínica. México: Manual Moderno.

Argollo, N., Bueno, O., Shayer, B., Godinho, K., Abreu, K., Durán, P., Assis, A., Lima, F., Silva, T., Guimarães, J., Carvalho, R., Moura, I., \& Seabra, A. G. (2009). Adaptação transcultural da Bateria NEPSY: avaliação neuropsicológica do desenvolvimento -estudo-piloto. Avaliação Psicológica, 8, 59-75.

Baddeley, A., \& Hitch, G. (1974). Working Memory. Em G. Bower (Ed.), The psychology of learning and motivation: advances in research and theory (pp. 47-89). Nova Iorque: Academic Press.

Baddeley, A., Anderson, M., \& Eysenck, M. (2011). Memória. (C. Stolting, trad.). Porto Alegre: Artmed. (Original publicado em 2009).

Baron, I. S. (2004). Neuropsychological evaluation of child. Nova Iorque: Oxford University Press.

Beeman, M. (1998). Coarse semantic coding and discourse comprehension. Em M. Beeman \& C. Chiarello (Eds.), Right hemisphere language comprehension: perspectives from cognitive neuroscience ( $\mathrm{pp}$. 255-284). Mahwah, NJ: Erlbaum.

Bentler, P. M. (2004). EQS 6 structural equations program manual. Encino, CA: Multivariate Software, Inc.
Brito, G. (1987). The Conners Abbreviated Teacher Rating Scale: development of norms in Brazil. Journal of Abnormal Child Psychology, 15, 511-518.

Crenitte, P. A. P., Batista, A. S., Silva, L., Lima, R. F., \& Ciasca, S. M. (2011). Estudo piloto de adaptação da Bateria Neuropsicológica Luria-Nebraska para Crianças (LNNB-C). Revista Psicopedagogia, 28, $117-25$

Fonseca, R. P., Casarin, F. S., Oliveira, C. R., Gindri, G., Ishigaki, E. C.S.S., Ortiz, K. Z., Parente, M. A. M. P., \& Scherer, L. C. (2011). Adaptação de instrumentos neuropsicológicos verbais: um fluxograma de procedimentos para além da tradução. Interação em Psicologia, 15(n. especial), 59-69.

Golden, C. J. (1987). Luria-Nebraska Neuropsychological Battery: children's revision-manual. Los Angeles: Western Psychological Services.

Hair, J. F., Black, W. C., Babin, B. J., Anderson, R. E., \& Tatham, R. L. (2009). Análise multivariada dos dados (6a ed.). Porto Alegre: Bookman.

Hershberger, S. L. (2003). The growth of structural equation modeling: 1994-2001. Structural Equation Modeling, 10, 35-46.

Korkman, M., Kirk, U., \& Kemp, S. (1998). NEPSY: a Developmental Neuropsychological Assessment. San Antonio, TX: The Psychological Corporation.

Krug, D., Dean, R., \& Anderson, J. (1995). Factor analysis of the Halstead-Reitan Neuropsychological Test Battery for Older Children. International Journal of Neuroscience, 83, 131-134.

Lezak, M. D., Howieson, D. B., \& Loring, D. W. (2004). Neuropsychological Assessment (4a ed.). Nova Iorque: Oxford University Press.

Livingston, R. B., Gray, R. M., Haak, R. A., \& Jennings, E. (1997). Factor structure of the Halstead-Reitan Neuropsychological Test Battery for Older Children. Child Neuropsychology, 3, 176-191.

Livingston, R., Gray, R., Haak, R., \& Jennings, E. (2000). Factor structure of the Reitan-Indiana Neuropsychological Battery for Children. Assessment, 7, 189-1997.

McCabe, D., Roediger, H., McDaniel, M., Balota, D., \& Hambrick, D. (2010). The relationship between working memory capacity and executive functioning: evidence for a common executive attention construct. Neuropsychology, 24, 222-243. 
McIntosh, A. R. (2007). Coding and representation: the importance of mesoscale dynamics. Em R. Roediger, Y. Dudai \& M. Fitzpatrick, Science of memory concepts. Nova Iorque: Oxford University Press.

Miranda, M. C., \& Muszkat, M. (2004). Neuropsicologia do desenvolvimento. Em V. M. Andrade, F. H. Santos \& O. F. A. Bueno (Eds.), Neuropsicologia hoje (pp. 211-224). São Paulo: Artes Médicas.

Mosconi, M., Nelson, L., \& Hooper, S. (2008). Confirmatory factor analysis of the NEPSY for younger and older school-age children. Psychological Reports, 102, 861-866.

Raven, J., Court, J., \& Raven, J. (1986). Colored progressive matrices. Londres: Lewis.

Reitan, R. M., \& Wolfson, D. (1992). Neuropsychological evaluation of young children. Tucson, AZ: Neuropsychology Press.

Salles, J. F., Fonseca, R. P., Miranda, M. C. Berlin, C., Rodrigues, C. C., \& Barbosa, T. (no prelo). Instrumento de Avaliação Neuropsicolinguística Breve Infantil NEUPSILIN-Inf. São Paulo: Vetor.

Salles, J. F., Fonseca, R. P., Rodrigues, C. C., Mello, C. B., Barbosa, T., \& Miranda, M. C. (2011). Desenvolvimento do Instrumento de Avaliação Neuropsicológica Breve Infantil NEUPSILIN-Inf. Psico-USF, 16, 297-305.

Scarf, D., Gross, J., Colombo, M., \& Hayne, H. (2011). To have and to hold: episodic memory in 3- and 4-year-old children. Developmental Psychobiology, n-a.

Schreiber, J. B., Stage, F. K., King, J., Nora, A., \& Barlow, E. A. (2006). Reporting structural equation modeling and confirmatory factor analysis results: a review. The Journal of Educational Research, 99, 324-337.

Semrud-Clikeman, M., \& Ellison, P. (2009). Child neuropsychology: assessment and interventions for neurodevelopmental disorders ( $2^{\mathrm{a}}$ ed.). Nova Iorque, NY: Springer Science and Business Media.

Simões, M. R. (2002). Avaliação neuropsicológica em crianças e adolescentes. Em R. Primi (Ed.), Temas em avaliação psicológica (pp. 26-34). Campinas: Instituto Brasileiro de Avaliação Psicológica.

Stinnett, T. A., Oehler-Stinnett, J., Fuqua, D., \& Palme, L. (2002). Examination of the underlying structure of the NEPSY: a developmental neuropsychological assessment. Journal of Psychoeducational Assessment, 20, 66-82.

Strauss, E., Sherman, E., \& Spreen, O. (2006). Compendium of neuropsychological tests: administration, norms, and commentary. New York: Oxford USA Trade.

Tulving, E., \& Szpunar, K. (2009). Episodic memory. Scholarpedia, 4, 3332.

Verdejo-García, A., \& Bechara, A. (2010). Neuropsicología de las funciones ejecutivas. Psicothema, 22, 227-235.

Wright, I., \& Sharples, P. M. (2008). Neuropsychological assessment in the neurology setting. Em J. Reed \& J. Warner-Rogers (Eds.), Child neuropsychology: concepts theory and practice (pp. 377-389). Oxford: Wiley Blackwell.

Recebido em: 08/04/2013

Reformulado em: 21/10/2013

Aprovado em: 12/11/2013 
Sobre os autores:

Jerusa Fumagalli de Salles é fonoaudióloga, mestre e doutora em Psicologia do Desenvolvimento pela Universidade Federal do Rio Grande do Sul (UFRGS), professora adjunta do Programa de Pós-Graduação em Psicologia da UFRGS e Coordenadora do Núcleo de Estudos em Neuropsicologia Cognitiva (NEUROCOG).

Juliana Burges Sbicigo é psicóloga pela Universidade do Vale do Rio dos Sinos, mestre e doutoranda em Psicologia pela Universidade Federal do Rio Grande do Sul e integra o Núcleo de Estudos em Neuropsicologia Cognitiva (NEUROCOG).

Wagner de Lara Machado é psicólogo pela Universidade Luterana do Brasil, mestre e doutorando em Psicologia pela Universidade Federal do Rio Grande do Sul, integra o Grupo de Estudo, Aplicação e Pesquisa em Avaliação Psicológica (GEAPAP/UFRGS).

Monica Carolina Miranda é psicóloga e neuropsicóloga, doutora em Ciências pelo Departamento de Psicobiologia da Universidade Federal de São Paulo - UNIFESP, pesquisadora-orientadora na UNIFESP e coordenadora do Núcleo de Atendimento Neuropsicológico Infantil (NANI).

Rochele Paz Fonseca é psicóloga e fonoaudióloga, mestre e doutora em Psicologia do Desenvolvimento pela Universidade Federal do Rio Grande do Sul, professora adjunta do Programa de Pós-Graduação em Psicologia da Pontifícia Universidade Católica do Rio Grande do Sul, pós-doutorada em Medicina (Radiologia) pela Universidade Federal do Rio de Janeiro e em Ciências Biomédicas pela Université de Montréal e coordenadora do Grupo de Neuropsicologia Clínica e Experimental (GNCE).

\section{Contato com os autores:}

Instituto de Psicologia, UFRGS

Avenida Ipiranga, 2.600, sala 114

90035-003 - Porto Alegre-RS. Brasil

E-mail: jerusafsalles@gmail.com 